\section{Frühes Mammakarzinom: duale HER2-Blockade}

Wie steht es um die Sicherheit und Effizienz einer dualen Blockade von HER2 mittels Pertuzumab und Trastuzumab in Kombination mit einer neoadjuvanten Chemotherapie beim frühen HER2-positiven Hochrisiko-Mammakarzinom?

\begin{abstract}
n der Phase-III-Studie GeparSepto wurde bei 1.206 Patientinnen mit primärem Hochrisiko-Mammakarzinom Standard-Paclitaxel in der sequentiellen neoadjuvanten Chemotherapie aus Paclitaxel, Epirubicin und Cyclophosphamid mit nab-Paclitaxel verglichen. Unter der nab-Paclitaxel-Kombination erreichten deutlich mehr Frauen eine pathologische Komplettremission (pCR). In der aktuellen Analyse wurden die Subgruppen der Patientinnen mit HER2-positiven Tumoren $(\mathrm{n}=396)$ und mit HER2-negativen Tumoren verglichen.

Die Teilnehmerinnen erhielten $4 \mathrm{Zy}$ klen wöchentliches Paclitaxel (je nach Randomisierung Standard-Paclitaxel oder nab-Paclitaxel) gefolgt von 4 Zyklen
\end{abstract}

Epirubicin plus Cyclophosphamid. Patientinnen mit HER2-positivem Tumor bekamen zusätzlich zur dualen HER2Blockade Trastuzumab plus Pertuzumab. Die Therapie mit Trastuzumab wurde noch 1 Jahr nach der Operation fortgesetzt. Primärer Endpunkt war die Rate an pCR, definiert als ypT0 ypN0.

Bei HER2-positiven Tumoren wurden höhere pCR-Raten erreicht als bei HER2-negativen Tumoren (57,8 vs. 22,0\%; $<<0,0001$ ), die höchste Rate in der Kohorte mit HER2-positiven, Hormonrezeptor(HR)-negativen Tumoren $(71,0 \%)$.

Nebenwirkungen vom Grad $\geq 3$ traten bei Patientinnen mit HER2-Positivität signifikant häufiger auf als bei Patientinnen mit HER2-Negativität, etwa Diarrhö vom Grad $3 / 4$ bei 7,6 vs. $0,9 \%$ ( $\mathrm{p}<0,001)$ und febrile Neutropenie vom Grad 3/4 bei 6,3 vs. $3,3 \%(p=0,023)$. Eine Abnahme der linksventrikulären Ejektionsfraktion (LVEF) gegenüber dem Ausgangswert kam häufiger bei Patientinnen mit HER2-positiven Tumoren vor (7,6 vs. 4,9\%). 2,0 bzw. 0,4\% der Patientinnen hatten einen LVEF-Rückgang auf weniger als $50 \%$ und um mindestens $10 \%$ gegenüber dem Ausgangswert. Eine Herzinsuffizienz (NYHA jeden Grades) trat bei 4 Patientinnen $(1,0 \%)$ der HER2-positiven Gruppe und bei $2(0,2 \%)$ der HER2-negativen Gruppe auf $(p=0,095)$.

Fazit: Beim frühen HER2-positiven Brustkrebs kann man mit einer dualen HER2-Blockade durch Pertuzumab und Trastuzumab in Kombination mit neoadjuvanter Chemotherapie hohe pCRRaten erreichen. Kathrin von Kieseritzky

Loibl $S$ et al. Dual HER2-blockade with pertuzumab and trastuzumab in HER2-positive early breast cancer: a subanalysis of data from the randomized phase III GeparSepto trial. Ann Oncol. 2016 Nov 9. [Epub ahead of print]

\section{Kardiovaskuläres Risiko der endokrinen Therapie bei Brustkrebspatientinnen}

\section{Kardiovaskuläre Erkrankungen sind eine der häufigsten Todesursachen älterer Brustkrebspatientinnen. Wie steht es um die kardiovaskuläre Sicherheit von Aromataseinhibitoren (Al) bei der endokrinen Therapie (ET)?}

$S_{\mathrm{E}}^{\mathrm{el}}$ it nachgewiesen wurde, dass AI in der ET des Mammakarzinoms gegenüber Tamoxifen (Tam) krankheitsfreies und Gesamtüberleben verlängern, sind sie bei postmenopausalen Patientinnen die bevorzugte Option. Die Aussicht auf eine mögliche sinnvolle Verlängerung der Therapie über die bisher empfohlenen 5 Jahre hinaus wirft auch die Frage nach der kardiovaskulären Sicherheit auf.

In der aktuellen bevölkerungsbasierten Beobachtungsstudie wurde die kardiovaskuläre Sicherheit von AI und Tam bei Frauen (> 55 Jahre) ermittelt, bei denen zwischen 2005 und 2010 Brustkrebs (Stadium I-III) diagnostiziert worden war. 7.409 Patientinnen wurden mit einem AI behandelt, 1.941 mit Tam. Das mediane Alter lag bei 71 bzw. 74 Jahren $(\mathrm{p}<0,001)$. Primärer Endpunkt war die Häufigkeit von Hospitalisierungen wegen eines Myokardinfarkts (MI). Um vor der Therapie bestehende Patientenunterschiede statistisch auszugleichen, nutzten die Forscher für einige Berechnungen eine Propensity-Score-Gewichtung.

Die Prävalenz ischämischer Herzkrankheiten war zu Beginn in beiden Gruppen vergleichbar (AI vs. Tam: 17,0 vs. $16,9 \% ; \mathrm{p}=0,96)$. In der Folge kam es zu 123 Klinikeinweisungen wegen akuter MI, 106-mal unter AI $(1,4 \%)$ und 17mal unter Tam (0,9\%). Die Myokardspezifische Hazard Ratio (HR), bei der die Tam-Gruppe als Referenz diente, war unter AI höher (HR 2,02, 95 \%-Kon- fidenzintervall 1,16-3,53). In den vordefinierten Subgruppen war eine ähnliche Verteilung zu beobachten.

Zusätzlich wurde die Mortalität analysiert: Unter Tam gab es während des Follow-ups (mittlere Dauer 1.146 Tage) 264 Todesfälle (Inzidenz 43,3 pro 1.000 Patientenjahre), in der AI-Gruppe 846 (34,7 pro 1.000 Patientenjahre). Damit war die Sterberate unter AI niedriger als unter Tam (HR 0,83; $p=0,014$ ). In einer Subgruppe mit geringerem kardiovaskulärem Risiko gab es zwischen den beiden Gruppen keinen signifikanten Unterschied in der Mortalität (HR 1,06; $\mathrm{p}=0,76$ ).

Fazit: AI sind in der Therapie des frühen Mammakarzinoms mit einem höheren Infarktrisiko assoziiert als Tam. Bei der individuellen Abwägung, welche ET jeweils angewendet werden soll, gilt es diesen Umstand zu berücksichtigen.

Kathrin von Kieseritzky

Abdel-Qadir $\mathrm{H}$ et al. The risk of myocardial infarction with aromatase inhibitors relative to tamoxifen in post-menopausal women with early stage breast cancer. Eur J Cancer. 2016;68:11-21. 